\title{
A RELAÇÃO ENTRE AUTISMO, FAMÍLIA E APRENDIZAGEM, EM ARTIGOS DA BASE DE DADOS SCIELO (2003-2019)
}

\section{LA RELACIÓN ENTRE AUTISMO, FAMILIA Y APRENDIZAJE, EN ARTÍCULOS DE LA BASE DE DATOS SCIELO (2003-2019)}

\author{
THE RELATION BETWEEN AUTISM, FAMILY AND LEARNING, IN ARTICLES \\ FROM THE SCIELO DATABASE (2003-2019)
}

\author{
Rafael Alexandre BELO ${ }^{1}$ \\ Thanyere Cavalcante da FONSECA ${ }^{2}$
}

RESUMO: Tem-se como norteador deste estudo a seguinte pergunta de pesquisa: Como a família influencia a aprendizagem de pessoas com Transtorno do Espectro Autista (TEA)? Foi realizada uma revisão de literatura integrativa na base de dados da Scielo (Scientific Electronic Library Online) com a combinação dos seguintes descritores: autismo; família; e aprendizagem. Obteve-se 21 artigos, entre pesquisas empíricas (13) e bibliográficas (8), que estabeleciam a seguinte relação com a pergunta norteadora: relação direta; relação indireta; relação enquanto pressuposto afirmativo. Os resultados indicam um consenso quanto a ser importante a participação da família em intervenções terapêuticas e pedagógicas. No entanto, percebe-se a necessidade de se superar a concepção unicamente biomédica, afim de abranger concepções sistêmicas e inclusivas, que concebam a família como protagonistas em realidades complexas.

PALAVRAS-CHAVE: Autismo. Família. Aprendizagem.

RESUMEN: Este estudio se guía por la siguiente pregunta de investigación: ¿Cómo influye la familia en el aprendizaje de las personas con trastorno del espectro autista (TEA)? Se realizó una revisión de literatura integradora en la base de datos Scielo (Scientific Electronic Library Online), mediante la combinación de los descriptores: autismo; familia; y aprendizaje. Se obtuvieron 21 artículos, incluyendo investigaciones empíricas (13) y bibliográficas (8), que establecieron la siguiente relación con la pregunta de investigación: relación directa; relación indirecta; relación de suposición afirmativa. Los resultados indican el consenso sobre la importación de la participación de la familia en intervenciones terapéuticas y pedagógicas. Sin embargo, se percibe una necesidad de superar una concepción unicamente biomédica, con el objetivo de considerar las concepciones sistémicas e inclusivas, que conciben la familia como protagonistas en realidades complejas.

PALABRAS CLAVE: Autismo. Familia. Arendizaje.

\footnotetext{
1 Universidade Federal de Alagoas (UFAL), Arapiraca - AL - Brasil. Professor do curso de Pedagogia. Doutorando em Educação (UFAL). ORCID: https://orcid.org/0000-0003-1230-1372. E-mail: rafaelbelo_paz@hotmail.com

${ }^{2}$ Associação dos Deficientes Físicos de Maribondo (ADEFIMAR), Marimbondo - AL - Brasil. Professora do Ensino Fundamental. Graduação em Pedagogia (UFAL). ORCID: https://orcid.org/0000-0001-6000-5741. E-mail: thanyerefonseca@gmail.com
} 
ABSTRACT: This study is guided by the following research question: How does the family influence the learning of people with Autism Spectrum Disorder (ASD)? Was carried out an integrative literature review in the Scielo database (Scientific Electronic Library Online), through of combination of the following descriptors: autism; family; and learning. We've obtained 21 articles, between empirical (13) and bibliographic researches (8), which established the following relation with the research question: direct relation; indirect relation; relation while affirmative assumption. The results are in accordance that family participation in therapeutic and pedagogical interventions is important. However, there is a need to overcome a biomedical conception, in order to include systemic and inclusive conceptions, which conceived the roles of the family as protagonists in complex realities.

KEYWORDS: Autism. Family. Learning.

\section{Introdução}

Atualmente, o autismo não é considerado uma doença, por não ter um caráter mórbido consumptivo que pode levar à algum tipo de degeneração física ou mesmo eventual morte. Esta perspectiva é reconhecida como o paradigma da neurodiversidade, que segundo Wuo (2019, p. 213) "vê o autismo como uma diferença que caracteriza a singularidade do sujeito e não uma doença".

Este modo de ver o autismo evita associa-lo a uma ideia de falta de saúde, embora, muitas vezes, a pessoa autista necessite de um trabalho de reabilitação que leve a um melhor convívio social e adequação às atividades acadêmicas, o que leva os profissionais de saúde ainda denominarem suas intervenções relacionadas ao autismo como 'tratamento'.

Destaca-se, ainda, que a concepção de doença se relaciona com aspectos sóciohistóricos amplos, ligados ao poder de medicalização da medicina.

Para efeitos de classificação contemporânea, a partir de 2013, com a quinta edição do Manual Diagnóstico e Estatístico de Transtornos Mentais - DSM-5, o Transtorno do Espectro Autista (TEA), passou a englobar o transtorno autista (autismo), o transtorno de Asperger, o transtorno desintegrativo da infância, o transtorno de Rett e o transtorno global do desenvolvimento sem outra especificação do DSM-IV, sendo caracterizado por déficits em dois domínios centrais: 1) déficits na comunicação social e interação social e 2) padrões repetitivos e restritos de comportamento, interesses e atividades. (AMERICAN PSYCHIATRIC ASSOCIATION, 2014).

O TEA também não é considerado uma deficiência, pois sua caracterização não se remete a nenhum déficit da capacidade cognitiva ou intelectual, embora existam casos que apresentem comorbidades. 
No entanto, para efeitos legais no Brasil, a partir da Lei n. 12.764 de 2012, a pessoa com TEA passou a ter os mesmos direitos da pessoa com deficiência. Implicando, por exemplo, na obrigatoriedade de as escolas regulares receberem a criança autista.

De acordo com Baña Castro (2015), foi devido a predominância do modelo clínicomédico que até pouco tempo o papel educador familiar não teve sua devida importância.

Estudo realizado por Guedes e Tada (2015), uma revisão da literatura científica sobre o autismo nos campos da Psicologia e da Educação, identificou o cenário da produção científica a partir de publicações em periódicos brasileiros entre de 2007 a 2012. Uma das categorias analisadas foi 'Autismo e relação familiares', que reuniu 18 artigos que abordavam temas ligados a: qualidade de vida do cuidador (9); impacto na dinâmica familiar (5); relação mãefilho (4).

No entanto, nenhum deles tratou especificamente a influência familiar no desenvolvimento e aprendizagem da pessoa autista, ainda que considerem a família "um suporte essencial para o desenvolvimento da pessoa com autismo junto às terapias multiprofissionais" (GUEDES; TADA, 2015, p. 306).

Assim, contribui-se para a temática através de uma revisão de literatura sobre a influência da família na aprendizagem e desenvolvimento de pessoas com TEA.

\section{Metodologia}

A presente revisão foi atualizada em janeiro de 2020, na base de dados da Scielo (Scientific Electronic Library Online), uma biblioteca eletrônica que disponibiliza artigos de revistas científicas nacionais e de mais outros 13 países.

A revisão de literatura foi realizada utilizando-se a abordagem da Integrativa, pois, sendo mais ampla, permite a inclusão de pesquisas de diferentes naturezas para a compreensão do fenômeno analisado.

Em síntese, utilizou-se os seguintes passos para a realização da revisão integrativa: elaboração do tema e pergunta norteadora; busca da amostragem na literatura, com estabelecimento de critérios de inclusão e exclusão; organização e seleção dos estudos; análise crítica; discussão dos resultados; apresentação da revisão.

Tendo como tema a influência da família na aprendizagem e desenvolvimento de crianças autistas, utilizou-se a seguinte pergunta norteadora: Como a família influencia a aprendizagem de pessoas com TEA?

Temas em Educ. e Saúde, Araraquara, v. 16, n. 1, p. 118-132, jan./jun., 2020. e-ISSN 2526-3471. 
Nesta pesquisa entendeu-se aprendizagem de forma genérica, considerando toda a aquisição de novos comportamentos, habilidades e conhecimentos que fazem parte do desenvolvimento humano, como a comunicação e a interação social.

O critério de inclusão neste estudo foi: artigos de pesquisas de variados procedimentos metodológicos, e áreas de estudo, que se posicionem em relação a influência da família na aprendizagem de pessoas autistas.

Foram excluídos estudos: que não versam sobre aprendizagem; que não versam sobre a família; que abordam o impacto de uma criança autista na família sem trazer reflexões sobre a aprendizagem e desenvolvimento; que na análise dos dados não especificam aqueles referentes às famílias com pessoas autistas.

Utilizou-se, as combinações dos descritores: 'autismo', 'família' e 'aprendizagem'. Com a combinação dos três descritores chegou-se à 5 artigos, que de forma direta ou indireta abordam a relação da família com o aprendizado de pessoas autistas. Posteriormente, utilizouse apenas os descritores 'autismo' e 'família', sendo levantados $59^{3}$ artigos, dos quais 18 foram selecionados. Por último, foram combinados os descritores 'autismo' e 'aprendizagem', sendo levantados $40^{4}$ artigos, dos quais 8 foram selecionados.

Considerando os artigos recorrentes nas combinações dos descritores, foram selecionados e analisados 21 artigos, publicados entre 2003 e 2019. Grande parte (10) publicada nos últimos três anos $(2017,2018$ e 2019), indicando um aumento recente das pesquisas que abordam de algum modo a influência da família no aprendizado de pessoas autistas. Quanto a origem da publicação: 17 são de periódicos brasileiros; 2 do Uruguai; 1 da Costa Rica; e 1 de Portugal.

O material selecionado foi classificado para análise quanto ao tipo de pesquisa: empírica (13), bibliográfica (4), bibliográfica do tipo revisão de literatura (4); e quanto a sua relação com a pergunta norteadora: relação direta (9), relação indireta (8), ou relação enquanto pressuposto afirmativo (4).

${ }^{3} \mathrm{O}$ banco de estudo acessado apresentou 62 artigos sendo que houve 3 duplicidades, o que resultou no quantitativo de 59 artigos.

${ }^{4} \mathrm{O}$ banco de estudo acessado apresentou 41 artigos sendo que houve 1 duplicidade, o que resultou no quantitativo de 40 artigos.

Temas em Educ. e Saúde, Araraquara, v. 16, n. 1, p. 118-132, jan./jun., 2020. e-ISSN 2526-3471. 


\section{Análise crítica}

Dos 21 artigos selecionados 4 apresentaram a influência da família no aprendizado da pessoa autista como pressuposto, ou seja, não como algo a ser respondido pela investigação, mas como um saber a priori que justificou a realização da mesma ou suas proposições.

Merlleti (2018) destaca a importância de cuidar daqueles que cuidam da criança, afirmando que são os pais quem dão a continuidade do tratamento.

Reis, Pereira e Almeida (2016) enfatizam que a família deve ser capacitada e corresponsabilizada para apoiar o desenvolvimento dos seus filhos e entender a importância da colaboração efetiva e constante entre família e profissionais para que os resultados das intervenções sejam alcançados.

Nem sempre a influência da família é apresentada como um pressuposto positivo. Assim, De Tilio (2017) em estudo realizado no estado de Minhas Gerais (Brasil) junto à irmã cuidadora de um adulto autista, apresenta o pressuposto que quando familiares/cuidadores não reconhecem as possibilidades de desenvolvimento (biológico e psicológico, incluindo a sexualidade), de modo a infantilizar, negar ou rechaçar, dificulta o aprendizado da pessoa autista em lidar com o próprio corpo e sua sexualidade.

Coelho, Iemma e Lopes-Herrera (2008, p. 80) considera o apoio da família um fator tão importante que questiona, em um estudo de caso realizado no interior de São Paulo (Brasil), “até que ponto a manutenção das características autísticas e os comportamentos apresentados pela criança estão sendo determinados pela privação sensorial que esta sofre, ou mesmo pela severidade do quadro autístico em questão".

Afirmar a influência da família implica em reconhecer a potencialidade que a pessoa autista possui, mesmo em relação aquelas habilidades que são desenvolvidas com maior dificuldade, como o caso da interação social.

A revisão de literatura realizada por Camargo e Bosa (2009) objetivou revisitar criticamente a literatura a respeito de competência social em estudos na área de autismo e inclusão escolar. Embora as autoras tenham considerado, na época, que havia poucos estudos sobre o tema e com limitações metodológicas, apontam a identificação de competência sociais em crianças autistas.

Entre as pesquisas relatadas, apresentam o estudo de $\operatorname{Serra}^{5}$ (2004, apud CAMARGO; BOSA, 2009, p. 70) que ao realizar o estudo com um menino autista com sete anos de idade,

${ }^{5}$ SERRA, D. C. G. A inclusão de uma criança com autismo na escola regular: desafios e processos. 2004.113 f. Dissertação (Mestrado) - Universidade do Estado do Rio de Janeiro, Rio de Janeiro, 2004.

Temas em Educ. e Saúde, Araraquara, v. 16, n. 1, p. 118-132, jan./jun., 2020. e-ISSN 2526-3471. 
acerca da inclusão escolar, constatou " [...] efeitos positivos da inclusão, na família, em função do maior investimento desta na aprendizagem da criança e um aumento na credibilidade nas potencialidades do filho".

Entre as pesquisas de cunho bibliográfico, destaca-se a comunicação do pesquisador, da universidade espanhola de Coruña, Baña Castro (2015), publicado na revista da universidade católica do Uruguai. Para o autor o suporte educativo da família é imprescindível, pois facilita o apoio e as oportunidades de desenvolvimento da pessoa com autismo para além de suas alterações e dificuldades.

Baña Castro (2015) apoia um posicionamento global e sistêmico, visando superar o modelo biomédico, ou seja, não focando no patológico e no déficit e sim em um corte educacional. Assim as necessidades e demandas do indivíduo e das famílias são consideradas, e a família deixa de ser uma mera prolongação passiva do trabalho dos profissionais.

A revisão de literatura realizada por Wuo (2019) em teses e dissertações nas regiões Sul e Sudeste do Brasil, entre 2008 e 2016, demonstrou que há ainda um predomínio das áreas médicas e, por consequência, vinculadas à noção de déficit e prejuízos psicológicos e sociais.

Reconhece-se, contudo, que a parti de 2008, com a Política Nacional de Educação Especial na Perspectiva da Educação Inclusiva, houve um aumento das pesquisas educacionais sobre o autismo, como alternativa a modelos exclusivamente médicos de olhar a diferença. Esta tendência, contribuiu para o desenvolvimento de um olhar sistêmico e tem colocado a família como um fator importantes em temáticas destacas por Wuo (2019) como: intervenção com familiares de pessoas com TEA; as relações escola-família no âmbito da inclusão escolar.

A ênfase numa perspectiva sistêmica, conforme defendida por Baña Castro (2015), mostra-se mais próxima de uma perspectiva inclusiva, uma vez que esta defende que os meios sociais, e as pessoas, em que o indivíduo está inserido possam ser transformados para que haja realmente uma inclusão genuína.

Já o estudo de Lampreia (2007) analisa alguns programas de intervenção precoce na perspectiva desenvolvimentista, que procura compreender os desvios do desenvolvimento da criança a partir do desenvolvimento típico. Ou seja, é uma abordagem próxima do modelo biomédico pois foca o déficit.

A identificação do autismo a partir dos 18 meses possibilita a intervenção precoce, que na perspectiva desenvolvimentistas possui característica pragmática e social, na qual, pressupondo a plasticidade cerebral, procura-se "estabelecer o caminho de desenvolvimento dos precursores da linguagem que não foi possível percorrer, independentemente da etiologia" 
(LAMPREIA, 2007, p. 106). Os pais tornarem-se coterapeutas é exigência para o sucesso dos programas, pois grande parte deles ocorre no ambiente familiar e diariamente.

Por outro lado, conclui-se que "são tantas as variáveis envolvidas que se torna praticamente inviável um maior controle experimental", de modo que "não se pode afirmar que as mudanças comportamentais observadas se devem à intervenção e não ao próprio desenvolvimento da criança” (LAMPREIA, 2007, p. 113).

Ainda sobre a intervenção precoce, Caldeira da Silva et al., (2003) descreve um programa clínico, desenvolvido em Portugal, baseado no modelo DIR (Developmental, Individual--difference, Relationship-based Model), presente também no estudo de Lampreia (2007).

De acordo com Caldeira da Silva et al. (2003, p. 32) "é um modelo de intervenção intensiva e global, que associa a abordagem Floor-time com o envolvimento e participação da família, com diferentes especialidades terapêuticas e a articulação e integração nas estruturas educacionais".

Caldeira da Silva et al., (2003, p. 37) conclui em seu estudo que "tanto pela clínica como pela adesão das famílias e dos técnicos, que este é um modelo útil e suficientemente eficaz para ser recomendada a sua aplicação e divulgação".

Observamos, contudo, que assim como acontece com os artigos revistos por Lampreia (2007), o artigo de Caldeira da Silva et al., (2003) apresenta uma descrição do programa sem, contudo, mencionar dados experimentais quanto à sua eficácia.

Em revisão de literatura sobre intervenções psicoeducacionais, Bosa (2006) identificou um crescente reconhecimento acerca da importância de envolver tanto as necessidades da criança autista como da família.

Para Bosa (2006) os resultados de pesquisa de intervenção nos casos de autismo devem ser interpretados com cautela, por ser grande o número de estudos que não são bem controlados metodologicamente. Além do mais, a autora avalia que "aparentemente, não existe uma única abordagem que seja totalmente eficaz para todas as crianças, em todas as diferentes etapas da vida" (BOSA, 2006, p. 52).

Lyra et al., (2017) realizou revisão de literatura no banco de dados de revisões sistemáticas Cochrane ${ }^{6}$ sobre intervenções para TEA. Dentre as 17 revisões consultadas nenhuma apresentou evidências fortes. Especificamente em relação às intervenções precoces

${ }^{6}$ Cochrane Libary é um banco de dados de colaboração internacional com revisões sistemáticas de pesquisas primárias em saúde, com alto rigor metodológico. Disponível em: www.cochranelibrary.com. Acesso em: dez. 2019. 
intermediadas pelos pais, as evidências foram consideradas fracas. Aspectos ligados à linguagem e comunicação, iniciação do filho na interação com os pais, comportamento adaptativo, trouxeram resultados inconclusivos e inconsistentes.

Houve, no entanto, evidência de mudança positiva e estaticamente significativa nos padrões de interação entre os pais e a criança, evidências sugestivas de melhora na compreensão da linguagem infantil e na redução da severidade das características do autismo infantil (LYRA et al., 2017).

A grande heterogeneidade de perfis das crianças e das famílias gera dificuldades de apropriação e controle de variáveis em contextos complexos e sistêmicos. Nesse sentido, Lampreia (2007) sugere que além de engajar os pais nos programas, deve-se considerar os estressores familiares e seu impacto sobre a criança.

Duas pesquisas empíricas selecionadas ajudam a discutir a complexidade em relação à dinâmica familiar: o estudo de Mapelli et al., (2018) e o de Sifuentes e Bosa (2010).

No estudo de Sifuentes e Bosa (2010) participaram 5 casais oriundos de Porto Alegre, com filhos com suposto diagnóstico de autismo e idade entre 4 e 7 anos. Analisou-se 4 dimensões do conceito de coparentalidade: a divisão de trabalho relacionado ao cuidado da criança, que compreende tarefas parentais como alimentação, organização de hábitos de higiene, transporte, auxílio em tarefas escolares e acompanhamento do filho em atividades recreativas; momentos de solidariedade e apoio; dissonância e antagonismo; e situações espontâneas dos pais para com a criança. Os resultados apontam que não há um compartilhamento igualitário das responsabilidades entre pai e mãe, apesar de existirem algumas divisões que seguem critérios bem pessoais, como afinidade, ou mesmo falta de tempo.

Verificou-se, também, que há uma sobrecarga de tarefas por parte da mãe e que uma das maiores fontes de conflito entre pai e mãe são divergências quanto às práticas educativas. (SIFUENTES; BOSA, 2010).

Já no estudo de Mapelli et al., (2018) contou-se com 22 participantes (pai, mãe, irmãos) de 15 famílias com crianças de até 12 anos incompletos, residentes no interior de São Paulo. Os resultados afirmam que o processo vivido pela família é dinâmico, e que em termos da influência na aprendizagem o estudo revelou o compromisso, sobretudo da figura materna, "em prover o melhor cuidado, inclusive em termos de estímulo ao desenvolvimento das potencialidades da criança" (MAPELLI et al., 2018, p. 6).

Sugere-se, ainda, que a esquipe de saúde deve compreender a família, sua estrutura e funcionamento, ou seja, compreender como ocorre a organização dessa família, suas relações e resiliências (MAPELLI, 2018).

Temas em Educ. e Saúde, Araraquara, v. 16, n. 1, p. 118-132, jan./jun., 2020. e-ISSN 2526-3471. 
Acerca de estudos com famílias que tenham um membro com TEA em fase adulta foram selecionados dois estudos, um deles, citado anteriormente, De Tilio (2017), fez o estudo apenas com uma familiar cuidadora. Já o estudo de Rosa, Matsukura e Squassoni (2019) realizou a aplicação de questionários eletrônicos à 67 familiares, em 14 estados brasileiros, ligadas a grupos ou instituições que trabalham com adultos autistas.

Apesar de não pesquisar diretamente a influência dos familiares na aprendizagem na pessoa autista, Rosa, Matsukura e Squassoni (2019, p. 313) sugerem que a participação da família foi fundamental durante a história dos sujeitos estudados, sobretudo para lidar com os desafios do processo de inclusão; além de reforçar a importância do compartilhamento entre escola e família quanto a responsabilidade de educar visualizando "as potencialidades e possibilidades reais" da criança com TEA.

Acerca da fonte de pesquisa, o estudo de Bialer (2017) se destaca por utilizar a autobiografia. A autora analisa os registros autobiográficos de uma família norte americana, publicados por sua mãe, cuja filha apresentou autismo severo. Foca-se os primeiros 15 anos de vida da biografada, retratando a evolução do quadro sintomático.

Bialer (2017, p. 1034) ressalta a importância da família, mais especificamente da intervenção da mãe (Clara) no desenvolvimento e aprendizagem da filha (Jessy). Embora Jessy parecesse em equilíbrio em seu mundo, isolada e "desconectada de tudo e de todos", a mãe compreendeu que este equilíbrio negava a possibilidade de crescimento.

Bialer (2017, p. 1028) destaca a postura da mãe:

Analisa todas as situações nas quais Jessy podia sair do isolamento, procurando recriar as condições que lhe viabilizavam sair do retraimento autístico. Concomitantemente, registrou áreas sensíveis nas quais não se devia pressioná-la sob risco de um retraimento reativo, realçando a importância do respeito ao seu tempo subjetivo.

Identificou-se seis pesquisas empíricas relacionados diretamente com a pergunta norteadora desta revisão de literatura: Menotti, Domeniconi e Benitez (2019); Balestro e Fernandes (2019); Cossio, Pereira e Rodriguez (2017); Rodrígues-Roblero (2015); Benitez e Domeniconi (2014); Fernandes et al., (2011).

O estudo de Menotti, Domeniconi e Benitez (2019) avaliou a eficácia de um pacote instrucional para o ensino de leitura de quinze palavras dissílabas (isoladas) para crianças com TEA, baseado no modelo de leitura como rede de relações. Participaram três crianças e seus pais, que foram envolvidos através da utilização de jogos, o que favoreceu a interação entre a díade e criou condições para a participação mais efetiva destes no processo escolar dos filhos. 
Os resultados indicam o que o procedimento favoreceu o aumento no repertório de leitura das crianças com TEA.

Com direcionamento semelhante, o estudo de Benitez e Domeniconi (2014) operacionalizou e avaliou uma capacitação de ensino de leitura e escrita para alunos da escola regular com deficiência intelectual e autistas. Incluiu-se 'professores da sala de aula regular', 'professores da educação especial' e os pais, de forma a vislumbrar o ensino compartilhado.

Os resultados indicam que a capacitação contribuiu "na operacionalização de estratégias inclusivas de ensino, de modo a atender os aprendizes com diferentes características, ritmos distintos de aprendizagem e potencialidades diferenciadas" (BENITEZ; DOMENICONI, 2014, p. 384).

Assim como Menotti, Domeniconi e Benitez (2019), Benitez e Domeniconi (2014, p. 381) sugerem que o "entrelaçamento" entre família e professores, como agentes educacionais, devam ser compreendidos como "estratégia promissora de ensino na perspectiva inclusiva".

Já o estudo de Cossio, Pereira e Rodrigues (2017) teve a finalidade de compreender os benefícios do apoio da intervenção precoce na percepção de seis mães de crianças com TEA, assim como a participação que consideram ter na intervenção. O estudo foi realizado em Portugal, país onde a intervenção precoce é regulamentada desde 2009 no Sistema Nacional de Intervenção Precoce na Infância (SNIPI).

Acerca dos benefícios da intervenção precoce no desenvolvimento dos filhos, as mães perceberam "melhoria na socialização, autonomia, memória, linguagem, motricidade, e ao nível do desfralde". Além disso, foi possível perceber "o empoderamento dos próprios pais quando estes criaram estratégias para potenciar as áreas de desenvolvimento dos filhos e adequaram a forma de lidar com as alterações ao nível do comportamento" (COSSIO; PEREIRA; RODRIGUEZ, 2017, p. 508).

Apesar dos dados qualitativos de relevância, referente a percepção das mães, o estudo de Cossio, Pereira e Rodriguez (2107) não apresenta dados experimentais quanto à eficácia do programa, do mesmo modo que o estudo de Caldeira da Silva et al., (2003) e os programas de intervenção precoce analisados por Lampreia (2007).

Para Balestro e Fernandes (2019) as intervenções de habilidades pragmáticas ${ }^{7}$ para crianças com TEA mais promissoras são aquelas que incluem a participação ativa da criança e dos seus cuidadores. Os autores analisaram a percepção dos cuidadores de 62 crianças com

${ }^{7}$ Como por exemplo as habilidades pragmáticas de comunicação, em que há a interligação dos aspectos sociais e emocionais utilizados em estratégias de interpretação, resolução de problemas, informações sociais e expectativas situacionais em contextos espontâneos (BALESTRO; FERNANDES, 2019).

Temas em Educ. e Saúde, Araraquara, v. 16, n. 1, p. 118-132, jan./jun., 2020. e-ISSN 2526-3471. 
TEA, no Rio Grande do Sul - Brasil, quanto ao perfil funcional da comunicação, e confirmaram a hipótese de que "seria possível produzir mudanças positivas na perspectiva com que pais de crianças com TEA percebem a comunicação de seus filhos a partir de um Programa de Orientações sobre Comunicação para Cuidadores (POCC) de crianças com TEA”.

Para as autoras, os resultados "demonstraram que o contexto, os comportamentos parentais atentos e responsivos, preveem subsequentes ganhos de linguagem em crianças com TEA" (BALESTRO; FERNANDES, 2019, p. 2).

Com perspectiva semelhante, o estudo de Fernandes et al., (2011) buscou verificar a interferência de orientações às mães de crianças autistas sobre os processos de comunicação e linguagem, perfil comunicativo e desempenho sociocognitivo; além de verificar a interferência das orientações sobre a forma como as mães observam as capacidades de seus filhos.

Com participação de 26 díades mãe-criança, os resultados mostram que todos os sujeitos apresentaram progresso em pelo menos uma das áreas investigadas, avaliando-se que "houve um impacto positivo de um procedimento de orientações sistematizadas, realizadas juntamente com o processo de terapia de linguagem das crianças (e não em substituição a ela)" (FERNANDES et al., 2011, p. 6).

Finalmente, a pesquisa-ação realizada por Rodríguez-Roblero (2015) trata de um processo pedagógico de um ano, ocorrido na Costa Rica, com um pai, uma professora e uma criança autista, estruturado em 12 sessões, utilizando-se jogos como instrumento de mediação.

A percepção da pesquisadora alcançou dimensões que geralmente são ocultadas nas ciências tradicionais de cunho mecanicista: “[...] en realidad el amor que le tiene el padre a su hijo es lo que logra cambios de actitud, respuestas que antes no se veían, expresiones, sonrisas, alegría" (RODRÍGUEZ-ROBLERO, 2015, p. 304).

O processo de mudança foi percebido em todos os participantes: "Todos estamos cambiando, el padre, el niño y yo" (RODRÍGUES-ROBLERO, 2015, p. 305). Por fim, o estudo evidenciou a importância da família no processo educativa não como simples receptores, e sim como protagonista.

\section{Considerações finais}

Nos artigos analisados há o consenso de que a participação da família de pessoas autistas é fundamental para o sucesso da intervenção terapêutica ou pedagógica. Em muitos artigos não selecionados a participação da família no sucesso da intervenção não foi mencionada, talvez 
por considera-la elementar. Mesmo assim, insistimos que, pela sua importância, o papel da família deve sempre ser enfatizado.

Além do que, como aponta Baña Castro (2015), é importante superar o modelo biomédico, que foca no patológico e no déficit, no caminho de uma visão sistêmica com um corte educacional. Assim a família deixa de ser apenas receptora dentro do processo educativo, para se tornar protagonista (RODRÍGUEZ-ROBLERO, 2015).

Estudos de base empírica (Menotti, Domeniconi e Benitez, 2019; Benitez e Comeniconi, 2014; Balestro e Fernandes, 2019; Fernandes et al., 2011) mostram que as possibilidades de aprendizagem da criança autista crescem quando há o envolvimento da família nas estratégias de intervenção.

Tendo em vista a análise realizada nesta revisão de literatura, concebemos que as potencialidades das famílias no âmbito da aprendizagem de pessoas autistas devem ser afirmadas, e consideradas um princípio elementar nas ações de saúde coletiva e educação inclusiva voltadas para este público.

Diante da diversidade possível da compreensão da família e sua dinâmica em realidades complexas, e pelo interesse que a temática traz para as diversas áreas de conhecimento que lidam com o autismo, sugerimos futuras pesquisas em perspectivas transdisciplinares sejam realizadas.

\section{REFERÊNCIAS}

AMERICAN PSYCHIATRIC ASSOCIATION (Org.). Manual diagnóstico e estatístico de transtornos mentais [recurso eletrônico]: DSM-5. Trad. Maria Inês Corrêa Nascimento. 5. ed. Porto Alegre: Artmed, 2014. Disponível em: http://aempreendedora.com.br/wpcontent/uploads/2017/04/Manual-Diagnóstico-e-Estatístico-de-Transtornos-Mentais-DSM5.pdf. Acesso em: 03 abr. 2018.

AZEVEDO, T. L.; CIA, F.; SPINAZOLA, C. C. Correlação entre o Relacionamento Conjugal, Rotina Familiar, Suporte Social, Necessidades e Qualidade de Vida de Pais e Mães de Crianças com Deficiência. Rev. bras. educ. espec., Bauru, v. 25, n. 2, p. 205-218, jun. 2019. Disponível em: https://www.scielo.br/scielo.php?script=sci_arttext\&pid=S141365382019000200205\&tlng=pt. Acesso em: 15 jan. 2020. DOI:

http://dx.doi.org/10.1590/s1413-65382519000200002

BALESTRO, J. I.; FERNANDES, F. D. M. Percepção de cuidadores de crianças com Transtorno do Espectro do Autismo quanto ao perfil comunicativo de seus filhos após um programa de orientação fonoaudiológica. CoDAS, São Paulo, v. 31, n. 1, e20170222, 2019. Disponível em: http://www.scielo.br/scielo.php?script=sci_arttext\&pid=S231717822019000100310\&lng=en\&nrm=iso. Acesso em: 15 jan. 2020. 
BANÃ CASTRO, M. El rol de la família em la calidad de vida y la autodeterminación de las personas com transtorno del espectro del autismo. Cienc. Psicol., Montevideo, v. 9, n. 2, p. 323-336, nov. 2015. Disponível em:

http://www.scielo.edu.uy/scielo.php?script=sci_arttext\&pid=S1688-

42212015000300009\&lng=es\&nrm=iso. Acesso em: 18 jan. 2020.

BENITEZ, P.; DOMENICONI, C. Capacitação de agentes educacionais: proposta de desenvolvimento de estratégias inclusivas. Rev. bras. educ. espec., Marília, v. 20, n. 3, p. 371-386, set. 2014. Disponível em:

http://www.scielo.br/scielo.php?script=sci_arttext\&pid=S1413-

65382014000300005\&lng=en\&nrm=iso. Acesso em: 18 jan. 2020.

BIALER, M. Um estudo descritivo do funcionamento psíquico de uma autista. Psicologia: Ciência e Profissão, [s.1.], v. 37, n. 4, p.1025-1036, dez. 2017. Disponível em:

http://www.scielo.br/scielo.php?script=sci_arttext\&pid=S1414-

98932017000401025\&lang=pt. Acesso em: 08 dez. 2018.

BOSA, C. A. Autismo: intervenções psicoeducacionais. Revista Brasileira de Psiquiatria, [s.1.], v. 28, n. 1, p. 47-53, maio 2006. Disponível em:

http://www.scielo.br/scielo.php?script=sci_arttext\&pid=S1516-44462006000500007. Acesso em: 08 dez. 2018.

CALDEIRA DA SILVA, P. et al. Programa clínico para o tratamento das perturbações da relação e da comunicação, baseado no Modelo D.I.R. Aná. Psicológica, Lisboa, v. 21, n. 1, p.31-39, jan. 2003. Disponível em:

http://www.scielo.mec.pt/scielo.php?script=sci_arttext\&pid=S0870-

$82312003000100005 \& \operatorname{lng}=$ pt\&nrm=iso. Acessos em: 24 jan. 2020.

CAMARGO, S. P. H.; BOSA, C. A. Competência social, inclusão escolar e autismo: revisão crítica da literatura. Psicol. Soc., Florianópolis, v. 21, n. 1, p.65-74, abr. 2009. Disponível em: http://www.scielo.br/scielo.php?script=sci_arttext\&pid=S0102-

$71822009000100008 \& \operatorname{lng}=e n \& n r m=i s o$. Acesso em: 14 jan. 2020.

COELHO, A. C. C.; IEMMA, E. P.; LOPES-HERRERA, S. A. Relato de caso: privação sensorial de estímulos e comportamentos autísticos. Rev. soc. bras. fonoaudiol., São Paulo, v. 13, n. 1, p. 75-81, mar. 2008. Disponível em:

http://www.scielo.br/scielo.php?script=sci_arttext\&pid=S1516-

$80342008000100013 \& \operatorname{lng}=e n \& n r m=i s o$. Acesso em: 23 jan. 2020.

COSSIO, A. do P.; PEREIRA, A. P. da S.; RODRIGUEZ, R. de C. C. Benefícios e nível de participação na intervenção precoce: perspectivas de mães de crianças com perturbação do espetro do autismo. Revista Brasileira de Educação Especial, Marília, v. 3, n. 4, p. 505-516, out. 2017. Disponível em: http://www.scielo.br/scielo.php?script=sci_abstract\&pid=S141365382017000400505\&lng=en\&nrm=iso\&tlng=pt. Acesso em: 08 dez. 2018.

CUERVO BOTERO, G. R. Los avatares del vínculo conyugal a la llegada de un hijo con autismo. Trab. soc., Bogotá, v. 21, n. 1, p. 169-193, jun. 2019. Disponível em:

http://www.scielo.org.co/scielo.php?script=sci_arttext\&pid=S2256-

54932019000100169\&lng=en\&nrm=iso. Acesso em: 15 jan. 2020. 
DE TILIO, R. Transtornos do espectro autista e sexualidade: um relato de caso na perspectiva do cuidador. Psicol. Conoc. Soc. [online], v. 7, n. 1, p.36-58, 2017. Disponível em:

$\mathrm{http}: / / \mathrm{www}$.scielo.edu.uy/scielo.php?script=sci_abstract\&pid=S1688-

$70262017000100036 \& \operatorname{lng}=e s \& n r m=i s o \& t \operatorname{lng}=$ pt. Acesso em: 15 jan. 2020.

FERNANDES, F. D. M. et al. Orientação a mães de crianças do espectro autístico a respeito da comunicação e linguagem. J. Soc. Bras. Fonoaudiol., São Paulo, v. 23, n. 1, p. 1-

7, mar. 2011. Disponível em: http://www.scielo.br/scielo.php?script=sci_arttext\&pid=S217964912011000100004\&lng=en\&nrm=iso. Acesso em: 18 jan. 2020.

GUEDES, N. P. da S.; TADA, I. N. C. A produção científica brasileira sobre autismo na psicologia e na educação. Psic.: Teor. e Pesq., Brasília, v. 31, n. 3, p. 303-309, set. 2015. Disponível em: http://www.scielo.br/scielo.php?script=sci_arttext\&pid=S0102$37722015000300303 \& \operatorname{lng}=$ en\&nrm=iso. Accesso em: 25 jan. 2020.

LAMPREIA, C. A perspectiva desenvolvimentista para a intervenção precoce no autismo.

Estud. psicol., Campinas, v. 24, n. 1, p. 105-114, mar. 2007. Disponível em:

https://www.scielo.br/scielo.php?script=sci_arttext\&pid=S0103-

166X2007000100012\&lng=pt\&tlng=pt. Acesso em: 23 jan. 2020. DOI:

http://dx.doi.org/10.1590/S0103-166X2007000100012

LYRA, L. et al. What do Cochrane systematic reviews say about interventions for autism spectrum disorders? São Paulo Med. Journal, São Paulo, v. 135, n. 2, p. 192-201, abr. 2017. Disponível em: http://www.scielo.br/pdf/spmj/v135n2/1806-9460-spmj-135-02-00192.pdf. Acessos em: 02 abr. 2019.

MAPELLI, L. D. et al. Child with autistic spectrum disorder: care from the family. Esc. Anna Nery, Rio de Janeiro, v. 22, n. 4, e20180116, 2018. Disponível em:

http://www.scielo.br/pdf/ean/v22n4/pt_1414-8145-ean-22-04-e20180116.pdf. Acesso em: 16 jan. 2020.

MENOTTI, A. R. S.; DOMENICONI, C.; BENITEZ, P. Atividades aplicadas pelos pais para ensinar leitura para filhos com autismo. Psicol. Esc. Educ., Maringá, v. 23, e185073, 2019. Disponível em: http://www.scielo.br/pdf/pee/v23/2175-3539-pee-23-e185073.pdf. Acesso em: 13 jan. 2020.

MERLLETI, C. Autismo em causa: historicidade diagnóstica, prática clínica e narrativas dos pais. Psicol. USP, São Paulo, v. 29, n. 1, p. 146-151, jan. 2018. Disponível em: http://www.scielo.br/pdf/pusp/v29n1/1678-5177-pusp-29-01-146.pdf. Acesso em: 08 dez. 2018.

REIS, H. I. S.; PEREIRA, A. P. S.; ALMEIDA, L. S. Características e especificidades da comunicação social na perturbação do espectro do autismo. Rev. bras. educ. espec., Marília, v. 22, n. 3, p.325-336, set. 2016. Disponível em: http://www.scielo.br/pdf/rbee/v22n3/14136538-rbee-22-03-0325.pdf. Acesso em: 14 jan. 2020.

RODRÍGUEZ-ROBLERO, M. del R. Trabajo conjunto familia y docente, el caso de un padre con su hijo autista: Una experiencia de investigación acción. Revista Electrónica Educare, [s.1.], v. 19, n. 1, p. 297-309, 1 jan. 2015. Disponível em:

https://www.scielo.sa.cr/pdf/ree/v19n1/a16v19n1.pdf. Acesso em: 08 dez. 2018. 
ROSA, F. D.; MATSUKURA, T. S.; SQUASSONI, C. E. Schooling of people with Autism Spectrum Disorder (ASD) in adulthood: reports and perspectives of parents and caregivers of adults with ASD. Cad. Bras. Ter. Ocup., São Carlos, v. 27, n. 2, p. 302-316, jun. 2019. Disponível em: http://www.scielo.br/pdf/cadbto/v27n2/2526-8910-cadbto-25268910ctoAO1845.pdf. Acesso em: 25 jan. 2020.

SIFUENTES, M.; BOSA, C. A. Criando pré-escolares com autismo: características e desafios da coparentalidade. Psicologia em Estudo, Maringá, v. 15, n. 3, p. 477-485, jul. 2010. Disponível em: http://www.scielo.br/pdf/pe/v15n3/v15n3a05.pdf. Acesso em: 08 dez. 2018.

WUO, Andrea Soares. Educação de pessoas com transtorno do espectro do autismo: estado do conhecimento em teses e dissertações nas regiões Sul e Sudeste do Brasil (2008-2016). Saúde soc., São Paulo, v. 28, n. 3, p. 210-223, set. 2019. Disponível em:

http://www.scielo.br/pdf/sausoc/v28n3/1984-0470-sausoc-28-03-210.pdf. Acesso em: 15 jan. 2020.

\section{Como referenciar este artigo}

BELO, Rafael Alexandre; FONSECA, Thanyere Cavalcante da. A relação entre autismo, família e aprendizagem, em artigos da base de dados Scielo (2003-2019). Temas em Educ. e Saúde, Araraquara, v. 16, n. 1, p. 118-132, jan./jun., 2020. e-ISSN 2526-3471. DOI: https://doi.org/10.26673/tes.v16i1.13539

Submetido em: 04/02/2020

Revisões requeridas: 10/04/2020

Aprovado em: 03/06/2020

Publicado em: 19/06/2020 